\title{
Research on EMI Reduction of Multi-stage Interleaved Bridgeless Power Factor Corrector
}

Li, Qingnan; Thomsen, Ole Cornelius; Andersen, Michael A. E.

Published in:

Asia-Pacific International Symposium on Electromagnetic Compatibility

Link to article, DOI:

10.1109/APEMC.2012.6237918

Publication date:

2012

Document Version

Publisher's PDF, also known as Version of record

Link back to DTU Orbit

Citation (APA):

Li, Q., Thomsen, O. C., \& Andersen, M. A. E. (2012). Research on EMI Reduction of Multi-stage Interleaved Bridgeless Power Factor Corrector. In Asia-Pacific International Symposium on Electromagnetic Compatibility (pp. 693-696). IEEE. https://doi.org/10.1109/APEMC.2012.6237918

\section{General rights}

Copyright and moral rights for the publications made accessible in the public portal are retained by the authors and/or other copyright owners and it is a condition of accessing publications that users recognise and abide by the legal requirements associated with these rights.

- Users may download and print one copy of any publication from the public portal for the purpose of private study or research.

- You may not further distribute the material or use it for any profit-making activity or commercial gain

- You may freely distribute the URL identifying the publication in the public portal 


\title{
Research on EMI Reduction of Multi-stage Interleaved Bridgeless Power Factor Corrector
}

\author{
Qingnan $\mathrm{Li}^{1}$, Ole C. Thomsen ${ }^{2}$, Michael A. E. Andersen ${ }^{2}$ \\ Department of Electrical Engineering, Technical University of Denmark \\ Oersteds Plads, Building 349, DK-2800 Kgs. Lyngby, DENMARK \\ ${ }^{1}$ ql@elektro.dtu.dk; ${ }^{2}$ oct@elektro.dtu.dk; ${ }^{2}$ ma@elektro.dtu.dk
}

\begin{abstract}
Working as an electronic pollution eliminator, the Power Factor Corrector's (PFC) own Electromagnetic Interference (EMI) problems have been blocking its performance improvement for long. In this paper, a systematic research on EMI generation of a multi-stage Two-Boost-Circuit Interleaved Bridgeless PFC (IBPFC) is presented. The insight into relationship of interleaving stages, switching on/off oscillations and EMI reduction is discussed. Finally, a $3.5 \mathrm{~kW}$ universal input 2-stage IBPFC prototype was built to verify the theoretical analysis. Experimental results show that significant EMI reductions at odd harmonics can be achieved by carefully designing the BPFC using interleaving technique.
\end{abstract}

\section{INTRODUCTION}

In order to develop the environment-friendly power electronics and reduce their pollutions to power grid, the active Power Factor Corrector (PFC) is always implemented in front of kinds of power converters. As one of the major optimal solutions of power quality improvement nowadays, the high efficient Bridgeless Power Factor Corrector (BPFC) have attracted lots of attentions [1-5]. For reducing the high electromagnetic pollution of BPFC due to fast switching on and off of the semiconductors, the interleaved BPFCs (IBPFCs) have recently been researched [6-8]. One possible architecture of IBPFC system is shown in Fig. 1, which is an extension version from Two-Boost-Circuit BPFC in Fig. 2. It is well-known that using interleaved technique, the EMI emission can be reduced. However, how much attenuation the BPFC can gain from interleaving stages is unclear. This work presents the insight into relationship of number of interleaved stages, switching on/off oscillations and EMI cancellation is discussed and proved by both simulation and experimental results. Furthermore, although the analysis is only based on Two-Boost-Circuit IBPFC, the idea can be extended to any other PFCs.

In the paper, the main advantages of IBPFC converters are firstly briefly introduced in Section II. In order to predict the EMI performances correctly, the common-mode (CM) and differential-mode (DM) EMI generation models for an $\mathrm{N}$ stage IBPFC are derived in Part A of Section III. The discussion on EMI reduction relates to number of interleaved stages and switching on/off oscillations is presented in Part B and $\mathrm{C}$ in Section III. Experimental results are carried out in Section IV. Finally, Section V comes to the conclusion.

\section{IBPFC INTRODUCTION}

The very popular BPFC topologies goes back to eighties [9]. References [3, 4] showed the basic performances of several well-known BPFC topologies. According to what has been proved in reference [4], compare to the traditional Boost PFC, one line frequency diode can be omitted in the current flowing path of BPFCs. Paper [4] also gave the detailed comparisons on EMI and semiconductor losses of five popular BPFCs and a conventional Boost PFC. In its conclusion, the Two-BoostCircuit BPFC in Fig. 2 shows better performances than others due to enhancing system efficiency without increasing EMI.

With the requirement of increasing power level, instead of simply paralleling several BPFCs together, during recent years, interleaved BPFCs is becoming more and more common. The latest publications [7] and [8] give the optimal design of a high efficient 2-stage IBPFC in Fig. 3. This novel topology can be expected to have better EMI performance comparing to the non-interleaved BPFC due to EMI cancellation.

Taking the multi-stage IBPFC in Fig. 1 as an example, the rest sections will give the detailed analysis on how do the interleaved stages and switching oscillations affect EMI cancellation in IBPFC systems.

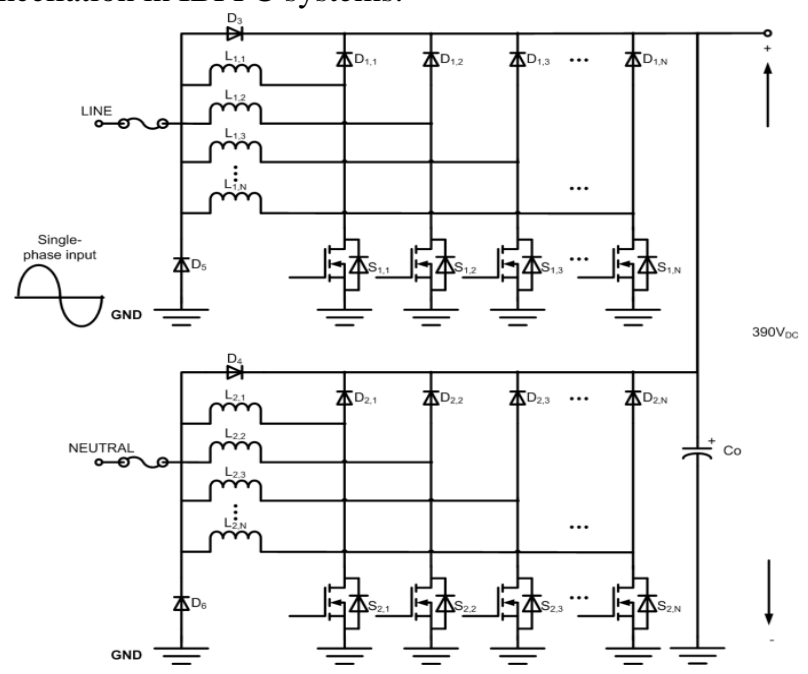

Fig. 1 Possible architecture of multi-stage IBPFC system

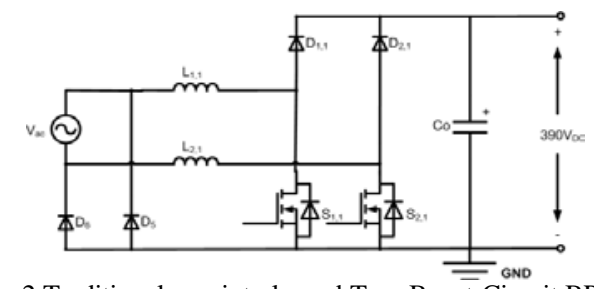

Fig. 2 Traditional non-interleaved Two-Boost-Circuit BPFC 


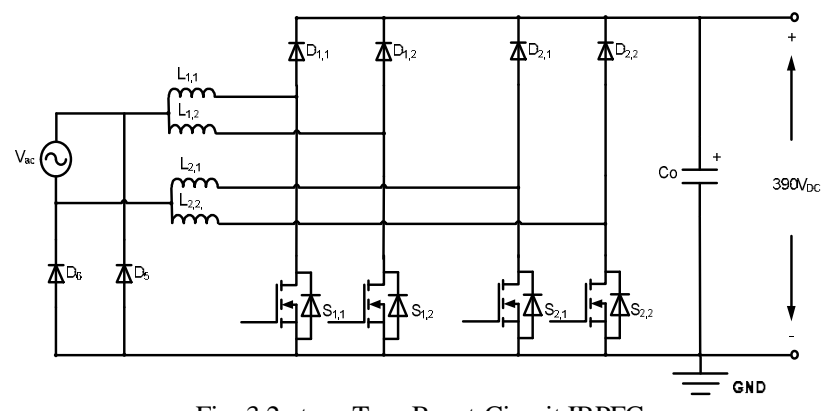

Fig. 3 2-stage Two-Boost-Circuit IBPFC

\section{MULTI-STAGE INTERLEAVED BPFC}

\section{A. EMI Noise Sources Modelling}

Because the $N$-stage interleaved BPFC (IBPFC) in Fig. 1 works symmetrically, only the positive ac period is considered here. In the positive half period, Boost inductors $\mathrm{L}_{1, i}$, MOSs $\mathrm{S}_{1, i}$ and Boost diodes $\mathrm{D}_{1, i}$ work interleaved with $\mathrm{D}_{6}$ returning current to $\mathrm{V}_{\mathrm{ac}}$. Where, $i$ symbolized the number of interleaved stages ranging from 1 to $N$. One of the major reasons for using IBPFC is EMI cancellation. However, since the PFC converter has variable on duty ratios, the high frequency ripple current can NOT cancel completely all the time [10]. In order to explain how exactly the EMI reduction varies with the number of interleaved stages increasing, it is better to analysis the CM and DM noises of the interleaved BPFC separately. However, it should be noticed that the real EMI performances typically depend strongly on the circuit layout, semiconductor characteristics, gate drivers, operating currents, voltages and temperature and parasitic elements. The actual EMI performances above $1 \mathrm{MHz}$ are very difficult to simulate. Therefore, in this paper, only the frequency noises below $1 \mathrm{MHz}$ are taken into consideration. Furthermore, the model of EMI receiver is not considered neither.

Start from the 2-stage IBPFC in Fig. 3, steps of DM EMI modeling of a multi-stage IBPFC is given in Fig. 4.

- $\quad$ Step 1: Using the symmetrical operation structure to simplify the topology.

- Step 2: Because the output filter capacitor can be considered as a short circuit in high frequency, and the return diode $\mathrm{D}_{6}$ is always on in the positive AC period, the two components can be ignored.

- Step 3: Simplify the semiconductor components and the Boost inductors. In high frequency domain, the Boost inductors charge and discharge through MOSs and Boost diodes, therefore, they can be considered as 2 triangle current sources.

- $\quad$ Step 4: Extend the DM EMI model from 2-stage to $\mathrm{N}$ stage by interleaving $N$ DM noise sources together. All the triangle current sources are $360^{\circ} / \mathrm{N}$ phase shift. Where, $N$ is the number of multi-stage interleaved modules. When $N$ equals to 1 , it symbolizes the noninterleaved BPFC.

Following the same steps, the CM model of the $N$-channel IBPFC is shown in Fig. 5.

\section{B. EMI Cancellation Analysis}

The mathematical derivation of the CM and DM noise sources in Fig. 4 and 5 are as follows. Assuming the $n$th order $\mathrm{CM}$ and $\mathrm{DM}$ noise of the first interleaving stage have a function of:

$$
\left\{\begin{array}{l}
V_{C M, 1}\left(n \omega_{o}\right)=A_{n} e^{j \varphi_{n}} \\
I_{D M, 1}\left(n \omega_{o}\right)=\frac{B_{n}}{N} e^{j \Phi_{n}}
\end{array}\right.
$$

Where, the $\omega_{o}$ is the fundamental angular frequency, $A_{n}$ and $B_{n}$ are the CM and DM harmonics' amplitudes of noninterleaved BPFC, and $\varphi_{n}$ and $\Phi_{n}$ are the initial phases of the $n$th harmonics. Therefore, the $n$th order CM and DM noise sources of the $N$ th interleaving stage are:

$$
\left\{\begin{array}{l}
V_{C M, N}\left(n \omega_{o}\right)=A_{n} e^{j\left(\varphi_{n}-\frac{2 \pi n(N-1)}{N}\right)} \\
I_{D M, N}\left(n \omega_{o}\right)=\frac{B_{n}}{N} e^{j\left(\phi_{n}-\frac{2 \pi n(N-1)}{N}\right)}
\end{array}\right.
$$

According to the superposition principle, the sum of the $n$th order CM and DM noises from the $N$-stage IBPFC can be expressed as:



Fig. 4 DM EMI modelling process of the $N$-stage Two-Boost-Circuit IBPFC

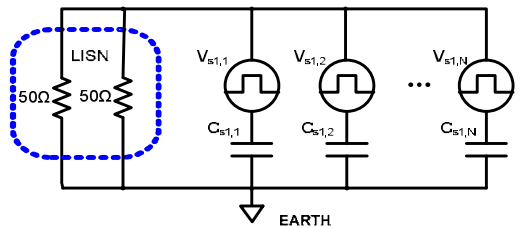

Fig. 5 CM EMI modelling of the $N$-stage Two-Boost-Circuit IBPFC 
Assuming $\Phi_{n}=\varphi_{n}=0$, Eqs. (3) and (4) can be simplified as:

$$
\begin{aligned}
& V_{C M, \text { tot }}\left(n \omega_{o}\right)=\frac{1}{N} \sum_{i=1}^{N} V_{C M, i}\left(n \omega_{o}\right) \\
& =\frac{A_{n}}{N}\left[\sum_{k=1}^{N} \cos \left(\frac{2 \pi n(k-1)}{N}\right)-j \sum_{k=1}^{N} \sin \left(\frac{2 \pi n(k-1)}{N}\right)\right] \\
& I_{D M, \text { tot }}\left(n \omega_{o}\right)=\sum_{i=1}^{N} I_{D M, i}\left(n \omega_{o}\right) \\
& =\frac{B_{n}}{N}\left[\sum_{k=1}^{N} \cos \left(\frac{2 \pi n(k-1)}{N}\right)-j \sum_{k=1}^{N} \sin \left(\frac{2 \pi n(k-1)}{N}\right)\right]
\end{aligned}
$$

From eqs. (1) and (5), the amplitude ratio of $n$th order CM noise of the $N$-stage interleaved and non-interleaved BPFC is:

$$
\begin{aligned}
& \alpha\left(n \omega_{o}\right)=\left|\frac{V_{C M, \text { tot }}\left(n \omega_{o}\right)}{V_{C M, n o n}\left(n \omega_{o}\right)}\right| \\
& =\frac{1}{N}\left|\sum_{k=1}^{N} \cos \left(\frac{2 \pi n(k-1)}{N}\right)-j \sum_{k=1}^{N} \sin \left(\frac{2 \pi n(k-1)}{N}\right)\right|
\end{aligned}
$$

similarly, from Eqs. (1) and (6), the amplitude ratio of $n$th order DM noise of the $N$-stage interleaved and non-interleaved $\mathrm{BPFC}$ is:

$$
\begin{aligned}
& \beta\left(n \omega_{o}\right)=\left|\frac{V_{D M, t o t}\left(n \omega_{o}\right)}{V_{D M, n o n}\left(n \omega_{o}\right)}\right| \\
& =\frac{1}{N}\left|\sum_{k=1}^{N} \cos \left(\frac{2 \pi n(k-1)}{N}\right)-j \sum_{k=1}^{N} \sin \left(\frac{2 \pi n(k-1)}{N}\right)\right|
\end{aligned}
$$

Where, $V_{C M, \text { tot. }}\left(n \omega_{0}\right)$ and $V_{D M, \text { tot. }}\left(n \omega_{0}\right)$ are the $n$th order CM and DM harmonics of the $N$-stage IBPFC; $V_{C M \text {,non. }}\left(n \omega_{o}\right)$ and $V_{D M, \text { non. }}\left(n \omega_{o}\right)$ are the $n$th order CM and DM harmonics of noninterleaved BPFC.

By using the Orthogonality principle:

$$
\left\{\begin{array}{l}
\alpha\left(n \omega_{o}\right)=\beta\left(n \omega_{o}\right)=0, n \neq c \cdot N \\
\alpha\left(n \omega_{o}\right)=\beta\left(n \omega_{o}\right)=1, n=c \cdot N
\end{array}\right.
$$

Where $c$ is the positive integer serials starts from 1 .

Hence, the amplitudes of high switching frequency noises from both CM and DM sources can be solved as below:

$$
\begin{gathered}
\left\{\begin{array}{c}
\left|V_{C M, t o t .}\left(n \omega_{o}\right)\right|=0, n \neq c \cdot N \\
\left|V_{C M, \text { tot. }}\left(n \omega_{o}\right)\right|=\left|V_{C M, \text { non }}\left(n \omega_{0}\right)\right|, n=c \cdot N
\end{array}\right. \\
\left\{\begin{array}{c}
\left|I_{D M, \text { tot } .}\left(n \omega_{0}\right)\right|=0, n \neq c \cdot N \\
\left|I_{D M, \text { tot }}\left(n \omega_{0}\right)\right|=\left|I_{D M, \text { non }}\left(n \omega_{0}\right)\right|, n=c \cdot N
\end{array}\right.
\end{gathered}
$$

From Eqs. (10) and (11), it can be concluded that:

1. Interleaved stages help to improve both CM and DM EMI.

2. In an $N$-stage IBPFC, except the $c \times N$ times fundamental frequencies, all the rest harmonics reduced due to the phase shift.

3. The more interleaved stages the IBPFC has, the more high frequency harmonics will be reduced.

4. The switching frequency $f_{s}$ affects the design of the EMI filter of IBPFC due to the noises cancellation do
NOT happen at $c \times N$ times fundamental frequencies. In order to maintain the EMI advantage of IBPFC, it is better to select its switching frequency based on the range of conduction EMI measurement. Implementing the European Standard EN55013, the disturbance voltage at mains terminals in the frequency range from $150 \mathrm{kHz}$ to $30 \mathrm{MHz}$ needs to be attenuated to fulfill the EMI limitation [11]. Therefore, the first harmonic of the IBPFC, which locates inside the range of the standard, should be better not to equal to $c \times N$ times of fundamental frequencies. The mathematic expression is:

$$
\left\lceil\frac{150 \mathrm{kHz}}{f_{s}}\right\rceil \neq c \cdot N
$$

Where $f_{s}$ is the switching frequency, and ' $\lceil x\rceil$ ' is the ceiling function, which returns the smallest integer not less than $x$. From Eq. (12) it can also be found, the IBPFCs usually require higher cut-off frequency of the EMI filter comparing to the non-interleaved one with the same EMI attenuations when $f_{s}$ is higher than $150 \mathrm{kHz}$.

Fig. 6 is the simulations of CM and DM EMI comparison between a 2-stage interleaved and a non-interleaved TwoBoost-Circuit BPFC, when the $f s$ is $75 \mathrm{kHz}$. Because the $2^{\text {nd }}$ harmonic $(150 \mathrm{kHz})$ is the first harmonic which locates inside the EMI standard's frequency range, and it cannot be cancelled by interleaving. (150kHz equals to the $c \times N$, when $c$ is 1 .) Therefore, the same as Eq. (12) has proved, the cut-off frequency of the EMI filter in this 2-stage IBPFC requires the same as the non-interleaved BPFC.

\section{Switching on/ off and Oscillation Analysis}

In the previous EMI models in part $B$, both of the rise/fall times and the oscillations of the switching are neglected. However, in the real power electronics systems, these effects normally cannot be avoided. Consider the oscillating drain to source single of MOS (the blue curve) in Fig. 7, and assume rise time equals to fall time. The real $V_{D S}^{\prime}$ signal can be defined with:

$$
V_{D S}^{\prime}(t)=\left\{\begin{array}{cl}
\frac{V_{D S}}{t_{r}} t, & 0 \leq t \leq t_{r} \\
V_{D S}+V_{p p} e^{-\gamma t} \sin \left[2 \pi f_{o}\left(t-t_{r}\right)\right], & t_{r}<t \leq \delta T_{s} \\
-\frac{V_{D S}}{t_{r}}\left(t-\delta T_{s}-t_{r}\right), & \delta T_{s}<t \leq \delta T_{s}+t_{r} \\
-V_{p p} e^{-\gamma\left(t-\delta T_{s}-t_{r}\right)} \sin \left[2 \pi f_{o}\left(t-t_{r}-\delta T_{s}\right)\right], \delta T_{s}+t_{r}<t \leq T_{s}
\end{array}\right.
$$
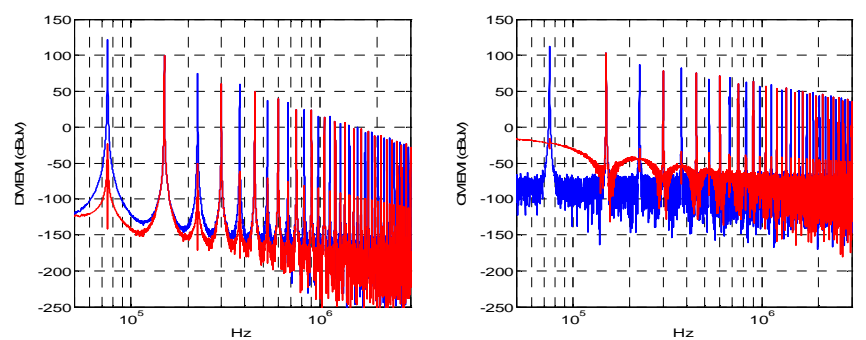

Fig. 6 DM (left) and CM (right) EMI comparison of 2-stage interleaved (red) and non-interleaved (blue) Two-Boost-Circuit BPFC at $75 \mathrm{kHz}$ and $3.5 \mathrm{~kW}$ 
Where $t_{r}$ is the switching rise and fall time, $V_{D S}$ is a $390 \mathrm{~V}$ constant drain to source voltage on MOS, $T_{S}$ is switching period, $V_{p p}$ is the oscillating peak voltage, $\gamma$ is attenuation factor of the oscillation, $\delta$ is MOS's on duty ratio and $f_{O}$ is the oscillating frequency. Implement Fast Fourier Transform (FFT), the frequency spectrums of Fig. 7 is shown in Fig. 8. From Fig. 8, it can be seen: the switching rise/fall times and oscillations rarely affect the EMI emission before the oscillating frequency and will not do harm to the low frequency EMI reduction caused by interleaved stages.

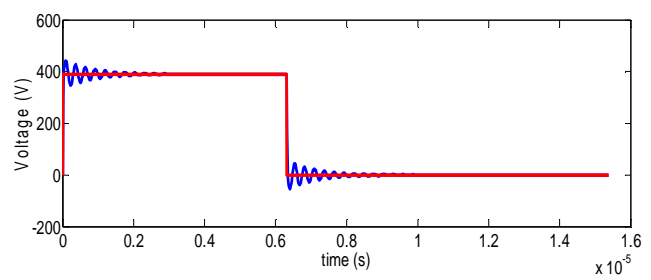

Fig. 7 Comparison of the oscillating $V_{D S}$ (blue) and the ideal $V_{D S}$ (red) when: $\mathrm{t}_{\mathrm{r}}=20 \mathrm{~ns} ; \delta=0.41 ; \quad \gamma=1.25 \mathrm{MHz} ; V_{p p}=0.15 V_{D s} ; f o=3.6 \mathrm{MHz}$.
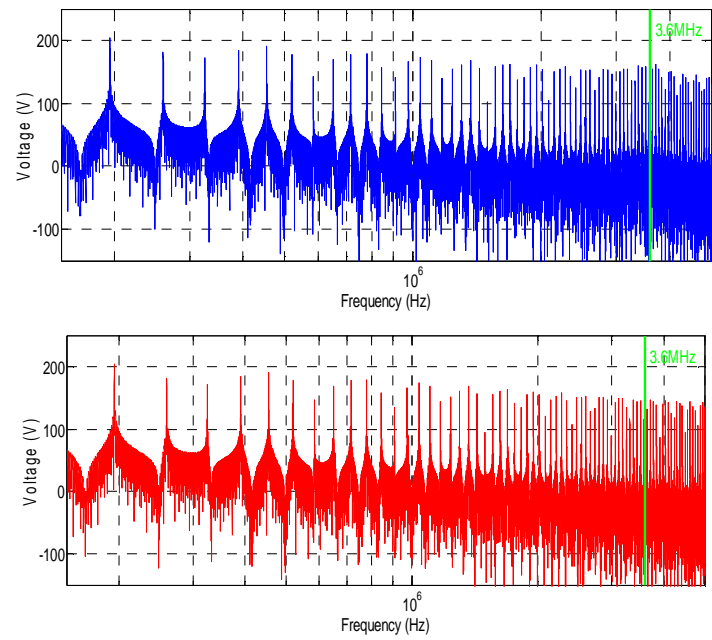

Fig. 8 Comparison of FFT of the oscillating $V_{D S}$ (blue) and the ideal $V_{D S}$ (red)

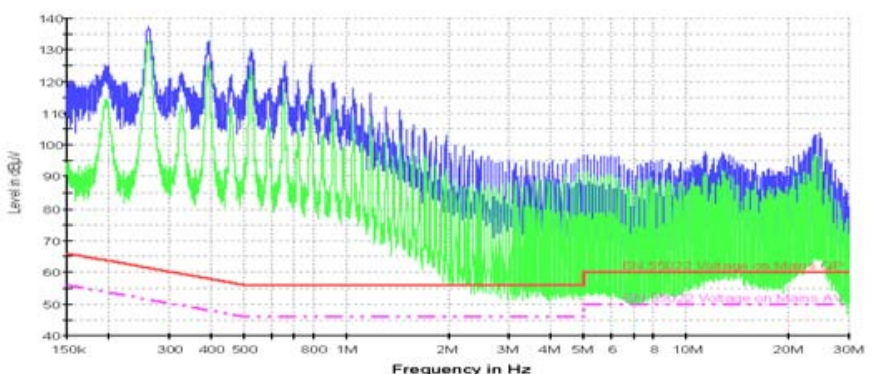

(a) 2-stage IBPFC

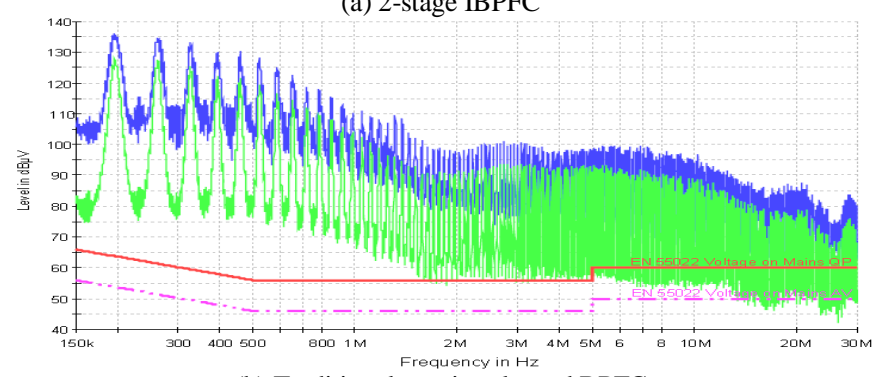

(b) Traditional non-interleaved BPFC

Fig. 9 EMI comparison of 2-stage IBPFC and non-interleaved BPFC at one eighth of full power and 230Vac input

\section{EXPERIMENTAL VERIFICATION}

Considering the design complexity and cost of multi-stage IBPFCs, a 2-stage $65 \mathrm{kHz} 390 \mathrm{Vdc}$ IBPFC for audio application was built for experimental verification. According to the special EMI requirement from audio systems, Fig. 9 gives the EMI measurement results of the 2-stage IBPFC and a traditional BPFC at one eighth of the full power. In order to make a fair comparison, all the components and testing equipments selected for the 2 topologies are the same. From Fig. 9, it is clear that in the 2-stage IBPFC, all the peak amplitudes of odd order harmonics have been reduced due to phase shift. However, according to what have been proved in Eqs. (10) and (11) in Section III, in the 2-stage IBPFC, all the odd harmonics should be completely cancelled, not only reduced. One reason for the difference between calculation and measurement is that: in the calculation, we don't consider the affect of EMI receiver. In the real measurement, the EMI receiver has a $9 \mathrm{kHz}$ resolution bandwidth (RBW) filter, which collects all the harmonics' amplitudes around the sweeping frequencies within the $9 \mathrm{kHz}$ bandwidth. So, it is impossible for the equipment to measure the exactly amplitude of any harmonic at one frequency point. But in the mathematical analysis in Section III, all the calculations relate to one frequency point. Anyhow, the 2-stage IBPFC still shows significant EMI reduction at odd harmonics.

\section{CONCLUSIONS}

In this paper, a systematic research on EMI generation of multi-stage IBPFC is presented by modeling its CM and DM noises. The insight into relationship of interleaving stages, switching on/off oscillations and EMI reduction based on the Two-Boost-Circuit IBPFC is discussed. Furthermore, a $3.5 \mathrm{~kW}$ universal input 2-stage IBPFC prototype was built and experimental results show that 10dB EMI reductions on odd order harmonics can be achieved by carefully designing the BPFC using interleaving technique.

\section{REFERENCES}

[1] H. Ye, Z. Yang, J. Dai, C. Yan, X. Xin, and J. Ying, "Common mode noise modeling and analysis of dual boost PFC circuit," in Proc. Int. Telecommunication Energy Conf., Sep. 2004, pp. 575-582.

pp. 575-582.
P. Kong, S.Wang, and F. C. Lee, "Common mode EMI noise suppression in bridgeless boost P. Kong, S.Wang, and F. C. Lee, "Common mode EMI noise suppression in bridg

L. Huber, Y. Jang, etc. "Performance evaluation of Bridgeless PFC Boost rectifiers" in: IEEE L. Huber, Y. Jang, etc. "Performance evaluation of Bridgeless
Transactions on Power Electronics, 2008, 23(3), pp. 1381-1390.

[4] Q. Li, M. A. E. Andersen and O. C. Thomsen, "Conduction losses and common mode EMI analysis on bridgeless power factor correction," in Proc. of International Conference on Powe Electronics and Drive Systems, Nov. 2009, pp. 1255-1260.

[5] Q. Li, M. A. E. Andersen and O. C. Thomsen, "Research on Power Factor Correction Boost Inductor Design Optimization - Efficiency vs. Power Density,” in Proc. of the $8^{\text {th }}$. International Conference on Power Electronics - ECCE (ICPE 2011-ECCE Asia), May 2011.

[6] B. Su and Z. Lu, "An interleaved Totem-pole Boost bridgeless rectifier with reduced reverserecovery problems for Power Factor Correction,” IEEE Trans. Power Electron., vol. 25, pp. 1406-1415, Jun. 2010.

[7] F. Musavi, W. Eberle and W. G. Dunford "A high performance single-phase bridgeless interleaved PFC converter for plug-in hybrid electric vehicle battery chargers," IEEE Trans. Industry Applications., vol. 47, pp. 1833-1843, Aug. 2011

[8] Q. Li, M. A. E. Andersen and O. C. Thomsen, "A Novel Bridgeless Power Factor Correction with Interleaved Boost Stages in Continous Current Mode," in Proc. of the 11th. International Aegean Conference on Electric Machines and Power Electronics and Electromotion Aegean Conference on Electric
(ACEMP2011), 2011, Sep. 2011.

[9] D. M. Mitchell, "AC-DC converter having an improved power factor," U.S. Patent 4412 277, Oct. $25,1983$.

[10] B. A. Miwa, D. M. Otten and M. E. Schlecht, "High efficiency power factor correction using interleaving techniques," Applied Power Electronics Conference, 1992, pp. 557-568, 1992.

[11] "Sound and televition broadcast receivers and associated equipment - radio disturbance characteristics - limits and method of measurement," BS EN 55013, 2001. 